\title{
Fast Solution of Electromagnetic Scattering Problems with Multiple Excitations Using the Recompressed Adaptive Cross Approximation
}

\author{
Mahdi Kazempour and Levent Gürel \\ Bilkent University Computational Electromagnetics Research Center (BiLCEM), Ankara, TR-06800, Turkey \\ lgurel@gmail.com
}

\begin{abstract}
We present an algebraic compression technique to accelerate the computation of multiple monostatic radar cross sections of arbitrary 3-D geometries. The method uses adaptive cross approximation, followed by a recompression technique to reduce the CPU time and the memory consumption. Each scattering problem due to a single excitation is solved with the multilevel fast multipole algorithm. The numerical results demonstrate the efficiency and accuracy of the proposed method.
\end{abstract}

\section{INTRODUCTION}

There are many circumstances where the knowledge of the radar cross section (RCS) of a target is crucially important. In some cases, measurement of the RCS of a target may not be possible or practical, leaving computation as the viable option. Since most radars rely on the backscattering from a target, computing the monostatic RCS (MRCS), where the incidence and observation directions are the same, is needed more often than the bistatic RCS. Computation of each MRCS value requires a separate solution, which may be costly depending on the size of the problem and the number of excitations. The task becomes considerably harder when the goal is to compute multiple MRCS values with high angular resolution [1].

Several methods are developed and used to speed up the solution of problems with multiple right-hand sides (RHSs). For instance, singular value decomposition (SVD) [2], interpolative decomposition (ID) [3], and adaptive cross approximation (ACA) [4] are algebraic methods that remove the redundancies in the initial excitation RHS matrix and result in a fast solution. Among these methods, ACA has two advantages: it has a low computational cost and, unlike SVD and ID, it does not require all elements of the excitation matrix, maintaining a relatively low level of memory consumption [5].

In this paper, we propose a recompression technique that incorporates SVD [6] on the matrices obtained from ACA to reach a more efficient low-rank decomposition of the RHS matrix. In other words, the main bottleneck of SVD, i.e., its high computational complexity, is solved by applying recompressed ACA (RACA) [7]. Due to the algebraic nature of the proposed method, it can be used in combination with all fast solvers [8], [9]. In this work, the solution of each excitation (corresponding to a single RHS) is accelerated by the multilevel fast multipole algorithm (MLFMA).

\section{IMPLEMENTATION}

The numerical solution of an electromagntic scattering problem using the method of moments (MoM) requires a discretization that yields a dense $N \times N$ matrix equation. To accelerate the solution, various methods are proposed that exploit the redundancies in the MoM matrix. One of the most efficient methods is MLFMA, which has a complexity of $\mathcal{O}(N \log N)$ [8]. Alternatively, there are purely algebraic methods. A subset of these methods is composed of solvers based on hierarchical matrices [9]. We use MLFMA as a fast solver for this work.

Although MLFMA may solve a problem with one RHS rapidly, solving multiple MRCS problems is time consuming. The matrix equation with an excitation matrix in the RHS can be represented as

$$
\overline{\boldsymbol{Z}}_{(N \times N)} \cdot \overline{\boldsymbol{X}}_{(N \times M)}=\overline{\boldsymbol{V}}_{(N \times M)},
$$

where $\bar{Z} \in \mathbb{C}^{N \times N}$ is the matrix of interactions between $N$ testing and basis functions, $\overline{\boldsymbol{X}} \in \mathbb{C}^{N \times M}$ contains the unknown coefficient vectors corresponding to the excitation vectors in the RHS matrix $\bar{V} \in \mathbb{C}^{N \times M}$, and $M$ is the number of excitations. The matrix $\overline{\boldsymbol{V}}_{(N \times M)}$ in (1) may contain linearly dependent columns. For a given threshold $\epsilon$, we use ACA to compress and factorize the RHS matrix. Applying ACA on $\bar{V}$ leads to

$$
\overline{\boldsymbol{V}}_{(N \times M)} \approx \overline{\boldsymbol{A}}_{(N \times k)} \cdot \overline{\boldsymbol{B}}_{(k \times M)}^{H},
$$

where $\overline{\boldsymbol{A}}_{(N \times k)} \in \mathbb{C}^{N \times k}, \overline{\boldsymbol{B}}_{(M \times k)} \in \mathbb{C}^{M \times k}$, and $k$ denotes the effective rank of $\overline{\boldsymbol{V}}$. To obtain a decomposition of $\overline{\boldsymbol{V}}$ with an effective rank $k^{\prime}\left(k^{\prime}<k\right)$, we employ a recompression technique that incorporates the $\mathrm{QR}$ decomposition and computes SVD in an efficient manner [6]. We apply a QR factorization for $\overline{\boldsymbol{A}}$ to get $\overline{\boldsymbol{A}}_{(N \times k)}=\overline{\boldsymbol{Q}}_{A(N \times k)} \cdot \overline{\boldsymbol{R}}_{A(k \times k)}$ and for $\overline{\boldsymbol{B}}$ to obtain $\overline{\boldsymbol{B}}_{(M \times k)}=\overline{\boldsymbol{Q}}_{B(M \times k)} \cdot \overline{\boldsymbol{R}}_{B(k \times k)}$, where matrices $\overline{\boldsymbol{Q}}_{A}$ and $\overline{\boldsymbol{Q}}_{B}$ are unitary matrices. Then, we perform a truncated SVD with a given threshold $\epsilon$ on the product of $\overline{\boldsymbol{R}}_{A}$ and $\overline{\boldsymbol{R}}_{B}^{H}$. After this recompression, the final low-rank approximation will be in the form of

$$
\overline{\boldsymbol{V}}_{(N \times M)} \approx \overline{\boldsymbol{A}}_{\left(N \times k^{\prime}\right)}^{\mathrm{RACA}} \cdot\left(\overline{\boldsymbol{B}}_{\left(k^{\prime} \times M\right)}^{\mathrm{RACA}}\right)^{H},
$$

where $k^{\prime}$ is the effective rank of the RHS matrix and is smaller than the rank $k$ achieved by ACA. An approximate solution 
to (1) can be achieved by substituting (3) in (1) and rewriting it as

$$
\overline{\boldsymbol{X}} \approx\left(\overline{\boldsymbol{Z}}^{-1} \cdot \overline{\boldsymbol{A}}\right) \cdot \overline{\boldsymbol{B}}^{H}
$$

where $\bar{Z}^{-1}$ denotes a standard solution, i.e., MLFMA in this work, but other fast methods can also be used to accelerate the solution.

\section{NUMERICAL RESULTS}

In this section, we investigate the 2-D MRCS values of the Flamme geometry at a frequency of $4 \mathrm{GHz}$. As shown in Fig. 1, the Flamme has a maximum length of $0.6 \mathrm{~m}$. The surface of the geometry is discretized with planar triangles with an average mesh size of $\lambda / 10$, which leads to 13,386 unknowns. The Flamme geometry is illuminated

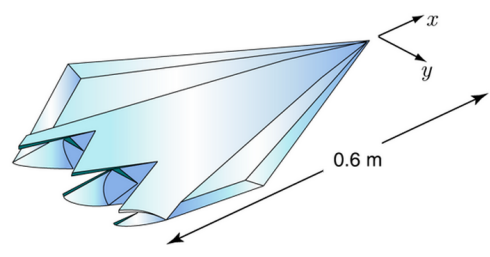

Fig. 1. Flamme geometry. The electrical size of the largest dimension of the geometry is $8 \lambda$ at $4 \mathrm{GHz}$.

with $\theta$-polarized plane waves incident from an angular sector defined by $\left[\theta_{\min }, \theta_{\max }\right]=\left[30^{\circ}, 50^{\circ}\right]$ and $\left[\phi_{\min }, \phi_{\max }\right]=$ $\left[45^{\circ}, 65^{\circ}\right]$. The angular resolution is $0.5^{\circ}$. The brute-force (BF) simulations require 1681 runs. Figure 2 illustrates multiple 2-D MRCS results. The results obtained from RACA are illustrated in Fig. 2(a). The relative error between the proposed method (RACA) and $\mathrm{BF}$ runs is calculated via $100 \times\left|\sigma_{\mathrm{BF}}-\sigma_{\mathrm{RACA}}\right| /\left|\sigma_{\mathrm{BF}}\right|_{\max }$ and illustrated in the Fig. 2(c). The selected resolution of spherical incident angles, i.e., (a) 2-D MRCS (RACA)

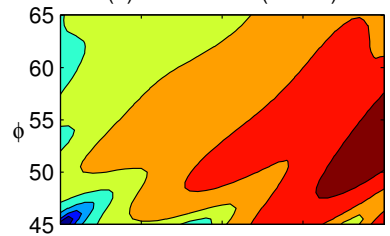

(c) Relative Error (RACA)

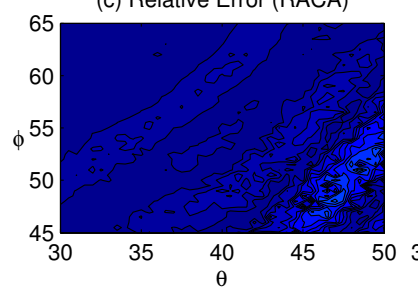

(b) 2-D MRCS (CS)

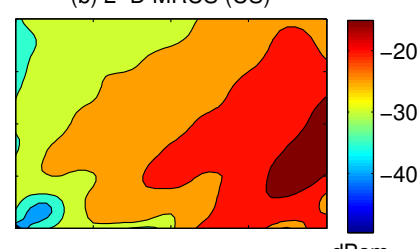

(d) Relative Error (CS)

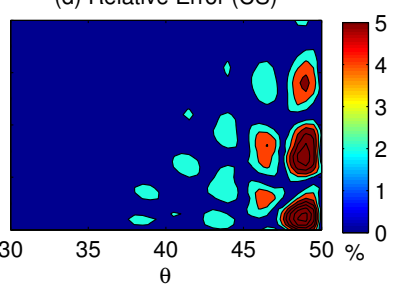

Fig. 2. $\theta \theta$-polarized 2-D MRCS of the Flamme. (a) 2-D MRCS obtained from the RACA. (b) 2-D MRCS computed using the CS interpolation. (c) The relative error of the RACA. (d) The relative error of the CS interpolation.

$\Delta \theta=\Delta \phi=0.5^{\circ}$, is less than the Nyquist resolution (i.e., $\Delta \theta_{\max }=\Delta \phi_{\max }=\frac{\lambda}{2 d}=3.58^{\circ}$ ). As a consequence, the RHS matrix will have linear dependencies. Using ACA algorithm reduces the required solutions from 1681 to 43 ; the number of solutions can be further reduced to 32 by incorporating RACA. In other words, we have a compression rate of almost
$98 \%$ in the RHS matrix. Table I summarizes all statistics of this example, and as evident from the table, the simulation time is reduced from almost 10.5 hours to about 18 minutes. To analyze the efficiency of RACA, we use the cubic-spline (CS) interpolation method to obtain $0.5^{\circ}$ resolution from the $\mathrm{BF}$ runs performed with $2.5^{\circ}<3.58^{\circ}$ angular intervals. The required number of $\mathrm{BF}$ runs in this case is 81 . The results of the CS interpolation are provided in Fig. 2(b). As shown in Fig. 2(d), the error of the CS interpolation technique is much higher than the error of RACA. The CS interpolation method requires more runs and leads to a higher error rate that indicates the efficiency of the RACA.

TABLE I

Statistics of the Flamme Simulations

\begin{tabular}{|c|c|}
\hline Number of Unknowns & 13386 \\
\hline ACA \& SVD Threshold & $10^{-4}$ \\
\hline$k$ in ACA & 43 \\
\hline$k^{\prime}$ of RACA & 32 \\
\hline Time for BF Run (s) & $38,489.3$ \\
\hline Time for RACA Run (s) & $1,058.7$ \\
\hline Speed-up Factor & 36.3 \\
\hline
\end{tabular}

\section{CONCLUSION}

We propose an efficient and low-complexity algorithm based on RACA to accelerate the computation of multiple MRCS. The results show that RACA is more accurate and more efficient than interpolation techniques. The method incorporates the QR decomposition and computes SVD in an efficient manner. Due to the purely algebraic nature of the proposed method, it can be combined with any fast solver.

\section{ACKNOWLEDGEMENT}

This work was supported by the Scientific and Technical Research Council of Turkey (TUBITAK) under Research Grant 111E203, by Schlumberger-Doll Research (SDR), and by contracts from ASELSAN, Turkish Aerospace Industries (TAI), and the Undersecretariat for Defense Industries (SSM).

\section{REFERENCES}

[1] M. Skolnik, Radar Handbook, NewYork: McGraw-Hill, 1990.

[2] Z. Peng, M. Stephanson, and J.-F. Lee, "Fast computation of angular responses of large-scale three-dimensional electromagnetic wave scattering," IEEE Trans. Antennas Propag., vol. 58, no. 9, pp. 3004-3012, 2010.

[3] X.-M. Pan and X.-Q. Sheng, "Fast Computation of Two-Dimensional Spatial Electromagnetic Scattering from Large-Scale Targets," in Proc. Computational Electromagnetics Workshop, CEM'13, Izmir, Turkey, Aug. 2013.

[4] A. Schroder, H.-D. Brüns, and C. Schuster, "A hybrid approach for rapid computation of two-dimensional monostatic radar cross section problems with the multilevel fast multipole algorithm," IEEE Trans. Antennas Propag., vol. 60, no. 12, pp. 6058-6061, Dec. 2012.

[5] M. Bebendorf, "Approximation of boundary element matrices," Numer. Math., vol. 86, no. 4, pp. 565-589, 2000.

[6] G. H. Golub and C. F. Van Loan, Matrix Computations, 3rd ed., Baltimore, MD: John Hopkins University Press, 1996.

[7] M. Bebendorf, Hierarchical Matrices: A Means to Efficiently Solve Elliptic Boundary Value Problems, Berlin: Springer-Verlag, 2008.

[8] Ö. Ergül and L. Gürel, "A hierarchical partitioning strategy for an efcient parallelization of the multilevel fast multipole algorithm," IEEE Trans. Antennas Propag., vol. 57, no. 6, pp. 1740-1750, Jun. 2009.

[9] M. Kazempour and L. Gürel, "Solution of low-frequency electromagnetics problems using hierarchical matrices," in Proc. Computational Electromagnetics Workshop, CEM'13, pp. 9-10, Izmir, Turkey, Aug. 2013. 